International Journal of Wireless \& Mobile Networks (IJWMN) Vol. 4, No. 2, April 2012

\title{
REALIZATION OF 3D UNDERWATER WIRELESS SENSOR NETWORKS AND INFLUENCE OF OCEAN PARAMETERS ON NODE LOCATION ESTIMATION
}

\author{
Samedha S. Naik ${ }^{1}$, Manisha J. Nene ${ }^{2}$, \\ Department of Computer Engineering ${ }^{1}$ \\ Department of Applied Mathematics and Computer Engineering ${ }^{2}$ \\ Defence Institute of Advance Technology, Pune, India 411025 \\ cse10 samedha@diat.ac.in ${ }^{1}$ mjneneddiat.ac.in ${ }^{2}$
}

\begin{abstract}
Autonomous Under Water Sensor Networks UWSNs form distributed amorphous computing environments. This emerging technology will pay off the need of conventional large, expensive, individual Ocean monitoring equipment. Efficient resolution for an unreachable UWSN which includes failureprone nodes will require strategies that are as simple as possible in computations and local communications, to facilitate self-organization. In this paper, we propose a distributed self organizing localization algorithm for localization in 3 Dimensional 3D UWSN. Unlike in terrestrial positioning, Under Water UW networks experience various impediments. These hurdles are caused due to variation in different UW parameters, especially in the ocean. We propose an efficient localization technique for $3 D$ UW networks. Our proposed technique eliminates errors encountered during localization process. Further we study the effect of sound speed using our proposed localization algorithm and localization technique. The proposed localization technique is also analysed for anomaly caused due to erroneous depth which is calculated using pressure sensors. The simulated results are analysed to find the average error in calculated node location. The results show that this localization technique realized using our self organizing algorithm incurs less computational and communicational burden.
\end{abstract}

\section{KEYWORDS}

Self Organizing, Underwater Localization, 3 dimensional Under Water Sensor Network, pressure-depth relationship, sound speed.

\section{INTRODUCTION}

UWSNs are collection of large number of sensor nodes deployed in the ocean. These sensors emit acoustic waves to communicate with each other. The sensor nodes are collectively responsible for collecting the sensed information and then relay it to surface station floating on the surface of the sea. The networked sensors coordinate to perform distributed sensing of environmental phenomena over large scale of physical space and enable reliable monitoring and control in various applications. In certain location dependent application [1][3] such as detection, classification and tracking of sea targets, each sensor nodes should be aware of its accurate location.

The main advantage of using Under Water UW acoustic sensor networks is that conventional large, expensive, individual ocean monitoring equipment units can be replaced by relatively small and less expensive UW sensor nodes that are able to communicate with each other via acoustic signals. Localizing these underwater sensor nodes is one of the essential tasks for

DOI : 10.5121/ijwmn.2012.4209 
International Journal of Wireless \& Mobile Networks (IJWMN) Vol. 4, No. 2, April 2012

UWSNs. The accurate location information of sensor nodes can be used in data tagging, routing, and node tracking.

The process of finding accurate location of any sensor node in UWSN is called as localization. Localization is a must-do task to get useful location-aware data. Many localization techniques do exist for terrestrial wireless sensor networks, but these techniques cannot be directly applied to underwater scenario. In this paper, we concentrate on attaining accurate results for localization inspite of the various challenges faced in UWSN [14]. The main focus in this paper will be on distributed localization techniques to compute accurate location for nodes in 3D environments. Further we also analyse the results when communication errors are induced.

\section{SCENARIO FOR UNDERWATER LOCALIZATION}

\subsection{Background}

Underwater acoustic channels are characterized by harsh physical layer environments with stringent bandwidth limitations [17]. The variable speed of sound and the long propagation delays under water pose a unique set of challenges for localization in UWSN [10]. Radio Frequency RF can work at the most on the ocean surface but fails for underwater [1] hence RF is not preferred for underwater scenario. For UWSN acoustic communication is preferred over optical and RF communication. Following are the reasons why acoustic communication is preferred over RF and optical waves: RF waves can travel in sea only at extra low frequencies (30-300 Hz). Hence large antenna and high transmission power is required. Other reasons are limited bandwidth, propagation delay (5 orders of magnitude greater than on terrestrial), very high bit error rates and temporary loss of connectivity. Hence, message exchanges between submerged UWSN nodes and surface nodes needed for localization must be carried out using acoustic communications.

There are many other challenges faced by UWSNs. The underwater channel is severely impaired, especially due to multi-path and fading. Battery power is limited and usually batteries cannot be recharged. Solar energy cannot be exploited. The issue of energy efficiency and the optimal data packet size/length in underwater wireless network communications in the context of effective and efficient data transmission is highlighted in [15]. UW sensors are prone to failures due of fouling and corrosion. Sensor nodes have very limited storage capacity. UW sensors may need to be able to do some data caching as the underwater channel may be intermittent and while the readings from UW sensors are often correlated. Spatial correlation is more unlikely to happen in underwater networks due to the higher distance among sensors. Unfortunately, underwater acoustic channels are characterized by long propagation delays, limited bandwidth, motion-induced Doppler shift, phase and amplitude fluctuations, multipath interference, etc.

To overcome the above challenges in UW scenario, few architectures and localization methods proposed are surveyed [2]. Most of these methods concentrate on localization based on the architectural behaviour (Anchor node positioning / mobility) or network behaviour (Centralized / Decentralized). Our algorithm, apart from being distributed in 3D, extends to improve over the results obtained during trilateration method.

\subsection{Underwater Sensor Network Architecture}

Different UWSN architectures are very well described in [1]. UW architectures can be classified based on their spatial coverage such as 2 Dimensional 2D/3D and node motion ability i.e. mobile/stationary/hybrid nodes. The 2D and 3D architectures can be either static or mobile. 
International Journal of Wireless \& Mobile Networks (IJWMN) Vol. 4, No. 2, April 2012

Several types of Autonomous Underwater Vehicles (AUVs) can be used to enhance the capabilities of underwater sensor networks. These vehicles are self propelled and move around the network to share data.

In $2 \mathrm{D}$ architecture, sensor nodes are placed at a same level i.e. all the sensor nodes have same depth, for example, sensors anchored at the bottom of the ocean. Each of these sensor node make use of acoustic transceivers to communicate with each other. In 3D architecture sensor nodes float at different depths. The two possible solutions for placing sensors at intermediate depths in the ocean can be: Attaching the sensor nodes to a surface buoy with a wire whose length can be adjusted. Another option is to adjust the length of the wire connecting the anchored nodes and the anchor.

AUV aided sensor networks though costly, can provide a better option to function without tethers, cables, or remote control. In [5] the localization algorithm for 2D UWSN is well explored. In section 4, we will be formulating localization algorithm for 3D UWSN.

\section{RELATED WORK}

Localization algorithms can be either classified as range based or range free. All the range based localization algorithms usually make use of different range measurement techniques. The four basic range measurement techniques are Received Signal Strength RSS, angle of Arrival AOA, Time Of Arrival TOA and Time Difference Of Arrival TDOA[3][16]. Each of which has its own merits and demerits for using them into the ocean. Amongst the above mentioned distance estimation techniques TOA is the most suitable for underwater scenario [4]. Reasons being that, the RSS algorithm is vulnerable to acoustic interferences, such as near-shore tide noise, nearsurface ship noise, multi-path, doppler frequency spread etc. The TDOA algorithm which use $\mathrm{RF}$ and acoustic signal is no longer feasible as the RF signal fails in underwater. The AOA algorithm requires directional transmission/reception devices, which would incur non-trivial extra cost. On the other hand, the TOA algorithm can be used in underwater environments measuring arrival time by using acoustic signal only. Hence TOA technique is suitable for UW distance calculation in our algorithm for UWSN deployment.

Recently large number of localization techniques has been proposed [2] most of them being range based and consider node mobility as their prime concern. We are interested in truly distributed algorithms that can be employed on large-scale ad-hoc sensor networks (100+ nodes). None of localization techniques IN [2] actually analyze localization error caused by underwater environment (such as temperature, pressure and salinity).

As discussed in the scenario in section 2, we will compare our technique with those in [2] which use ToA ranging and anchor nodes. Few of the techniques which are anchor based and use ToA range measurement are discussed here. We can group these techniques based on the major concern during localization.

MASL[18] (Motion-Aware Self-Localization for underwater networks) deals with the inaccuracy in distance estimation caused due to node movements. Whereas 3D-MALS[19], CL[20] (Collaborative localization for fleets of underwater drifters), DNRL[21] (Localization with Dive'N'Rise (DNR) Beacons for Underwater Acoustic Sensor Networks) and DETL[22] (A localization scheme for underwater wireless sensor networks) exercise movement of anchor nodes/sensor nodes through the water column to improve coverage.

Other techniques like AAL [23] (AUV-Aided Localization for Underwater Sensor Networks), LDB[24] (Localization with directional beacons for sparse 3d underwater sensor networks) and MSL[25] (Multi Stage Underwater Sensor Localization Using Mobile Beacons) make use of AUVs for localization. 
International Journal of Wireless \& Mobile Networks (IJWMN) Vol. 4, No. 2, April 2012

3DUL[27] (A three dimensional localization algorithm for underwater acoustic sensor networks) is an iterative process where it starts with the surface anchors (GPS driven) acting as known nodes and localizing the subsequent one distance UW sensor nodes. SLMP[28] (Scalable localization with mobility prediction for underwater sensor networks) is a technique for mobile UWSN, Anchor node estimation are done by using their previous coordinates and their mobility patters. LSHL[26] (Localization for large-scale underwater sensor networks) is a technique which use the same architecture as in our proposed technique, however we overcome the computational overhead by eliminating the need of computing extended Euclidean distance between two nodes.

To the best of our knowledge none of the work incorporates the impediments introduced due to UW parameters. Our localization technique realized using our proposed self-organizing algorithm, considers parameters like temperature, salinity and pressure while estimation of location value to the unknown node in UWSN.

\section{OUR WORK}

\subsection{Localization Scenario}

In our proposal we consider the requirement for underwater sensor networks to be selforganizing which implies that there is no central control to control randomly deployed UWSN. Consequently, we assume that nodes are randomly distributed across the environment. Nodes are dropped into the ocean either by plane or ship. Once they settle on the sea floor they start communicating to each other using acoustic signals. The sensors must then estimate their position using an efficient positioning algorithm. The proposed algorithm does not rely on any existence of previous infrastructure. At present we assume that there are mild water currents. Deployed large scaled UW sensors include nodes called Reference Nodes RN and Ordinary Nodes OrN. RNs are able to detect their position by means of GPS, which is attained before diving into the ocean. These RNs play an important role in finding the accurate location of OrNs. OrNs are those nodes sunk underwater which are location unaware. The RN, apart from being positioned at a single location in a network, can be made to rise and dive in the water column to share its location with other OrNs encountered on its way [18][21][29]. In order to perform collaborative sensing tasks the sensor nodes must estimate their position by means of a distributed positioning algorithm. Our proposed algorithm is fully distributed and will be useful in such scenarios.

The communication architecture of underwater sensor networks constitute of OrNs that are anchored to the bottom of the ocean as shown in Figure 1. The depths of the OrNs are assumed to be variable, to form a 3D environment. The depth can be adjusted by adjusting the length of the cable connected to the anchors. Underwater sensor nodes are interconnected to one another by means of wireless acoustic links. Using acoustic communication the sensor nodes can relay data from the ocean bottom network to a surface station.

UW-sensors are equipped with two acoustic transceivers, namely a vertical and a horizontal transceiver. The horizontal transceiver is used to send commands and configuration data to the other sensors and the vertical link is used to relay data to a surface station. It is assumed that all the nodes have same communication range. Two types of sensor nodes are deployed (i). RNs / Anchor nodes / Beacons, these are the nodes which know their locations. (ii). OrNs are those sensor nodes UW which are unaware of their locations. Our proposed self-localization algorithm executes without any centralized control with an aim to make randomly deployed UWSN to be location-aware. 


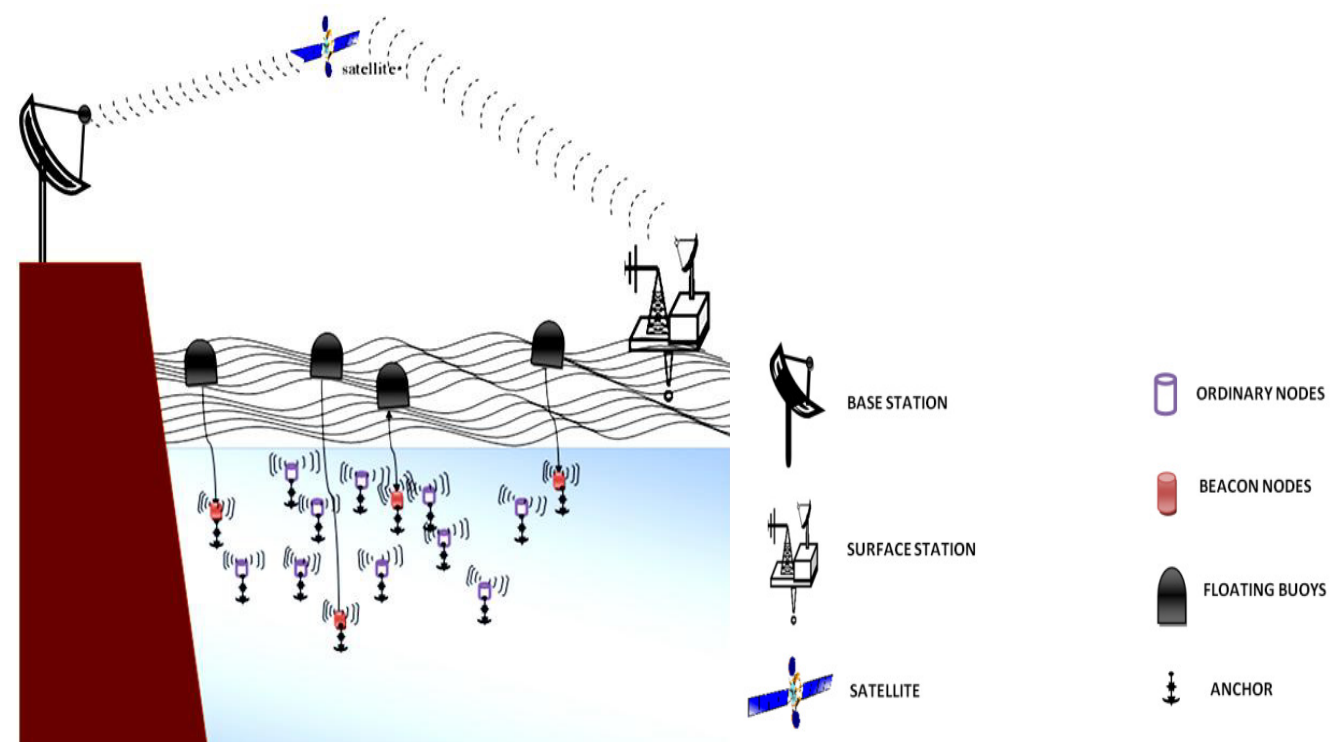

Figure 1. Deployment Scenario for UWSN

\subsection{Motivation}

UWSN localization with 3D architecture may be more tricky than with 2D architecture. In 2D architectures, the sensing coverage will be only in a particular plane, thereby restricting itself to scan only the plane covered by the nodes.

Every node in an UWSN communicates using acoustic signals. These signals experience propagation delay because of the ocean parameters like Pressure, Temperature, Salinity and Altitude. While devising an efficient localization algorithm it becomes very crucial to study the impact of above parameters on the algorithm.

In 3D UWSN, out of the three coordinates $(x, y, z)$, one of the coordinate i.e. depth can be found by a pressure sensor. Finding the Depth of a node becomes much easier by using pressure sensors. But at the same time we cannot neglect the errors encountered during depth calculation. The detailed calculation will be shown in section 6.2.

\subsection{Our contribution}

We propose a novel self-organizing localization algorithm which autonomously performs the assigned task without human intervention using large scale UWSN.

The proposed localization technique uses only the distance estimation between the anchor and ordinary node. Computational and communication overheads are thus reduced. The effect of difference in depth, which is calculated by pressure sensors for each node, is considered by our proposed technique for location estimation of unknown nodes in 3D environment. Further the errors in distance estimation caused due to sound speed are included by our proposed localization technique which is realized by our proposed distributed self-organizing algorithm. The result of our proposed technique is simulated and analyzed in this research article. 


\section{Proposed algorithm}

We propose an adaptive self-organizing localization algorithm for UWSN. The sensor nodes are deployed randomly at varying depth in the ocean. Here the randomly deployed underwater sensor nodes self-localize to be location-aware. Our proposed localization algorithm for 3D environment is completely decentralized and distributed in nature.

Average Error AE is calculated to weigh the efficiency of our algorithm. The average error will be given as:

$\mathrm{AE}=\frac{\sum_{\mathrm{i}=1}^{500} \sqrt{\left(\left(\mathrm{x}_{\mathrm{i}}-\mathrm{x}_{\mathrm{i}}^{*}\right)^{2}+\left(\mathrm{y}_{\mathrm{i}}-\mathrm{y}_{\mathrm{i}}^{*}\right)^{2}+\left(\mathrm{z}_{\mathrm{i}}-\mathrm{z}_{\mathrm{i}}^{*}\right)^{2}\right)}}{500}$

where $\left(\mathrm{x}_{\mathrm{i}}, \mathrm{y}_{\mathrm{i}}\right)$ is a real sensor position and $\left(\mathrm{x}_{\mathrm{i}}{ }^{*}, \mathrm{y}_{\mathrm{i}}{ }^{*}\right)$ is estimated localization.

\subsection{Phases in proposed algorithm}

Proposed algorithm incorporates different phases which include deployment; distance estimation; initialization of RN's and position estimation. A Pictorial view of the proposed algorithm is presented in Figure.2.

\subsubsection{Node deployment}

Nodes are deployed in water by plane or by ship. Before deployment, the data structure: beacon_flags are reset for all the sensor node. The data structure: beacon_flag of RN is set to ' 1 '. The attributes of a sensor node are Node ID, Network ID, Beacon flag, list of reference nodes, its $\mathrm{x}, \mathrm{y}, \mathrm{z}$ position (where $\mathrm{z}$ is the depth) at which node is place. Node ID is a unique number which identifies a node. Network ID tells to which network this node belongs. Beacon Flag stores the status of a node i.e. whether it is an anchor or an ordinary node. If beacon flag is set to ' 1 ' it implies that the node is a beacon/anchor node and knows its location. If beacon flag is set to ' 0 ' the node is an ordinary node whose location is yet to be found out. Before deployment all the ordinary nodes are set to ' 0 ' and anchor nodes are set to ' 1 '. 
International Journal of Wireless \& Mobile Networks (IJWMN) Vol. 4, No. 2, April 2012

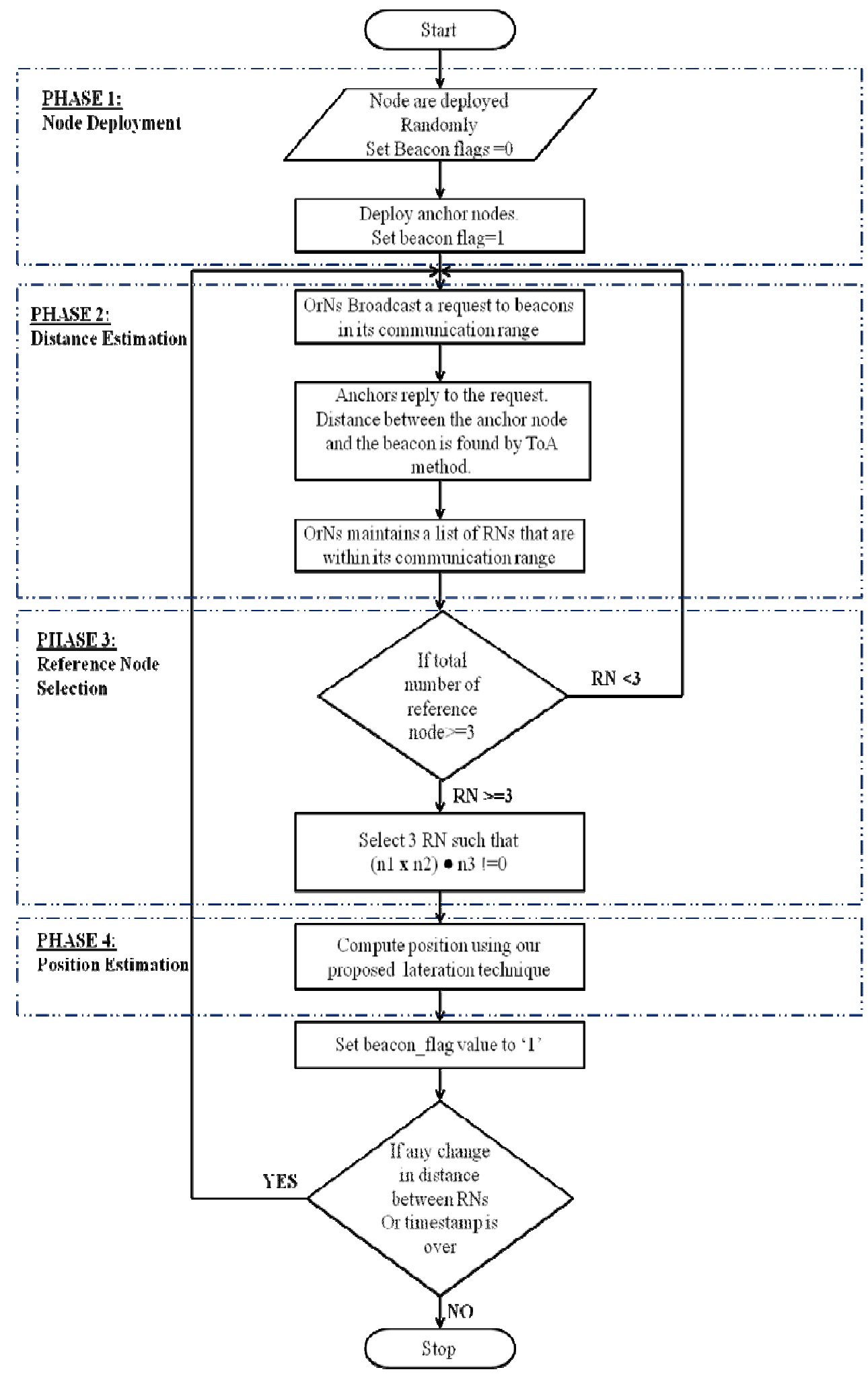

Figure 2. Flow Chart for Our Proposed Algorithm 


\subsubsection{Distance estimation}

In order to find the position of sensor node, minimum of three $\mathrm{RN}$ are needed. The distance between these RN are used to calculate the exact position of unknown node. In our proposed technique, distance estimation between the OrN and RN will be found by TOA method. For TOA-based systems, the one-way propagation time is measured, and the distance between measuring unit and signal transmitter is calculated. This algorithm estimates the distance between nodes by measuring the propagation time of a signal. So it requires precise time synchronization between two nodes. In this case the distance between two nodes is directly proportional to the time the signal takes to propagate from one point to another. If signal is sent at time $\mathrm{t} 1$ and reached the receiver node at time $\mathrm{t} 2$, the distance between two nodes can be defined as in equation (1). Where $\mathrm{Sr}$ is the propagation speed of acoustic signal $(1500 \mathrm{~m} / \mathrm{s})$. From this method we get the list of all possible RN's in the communication range.

$$
\mathrm{d} 1=\mathrm{Sr}(\mathrm{t} 1-\mathrm{t} 2)
$$

\subsubsection{Reference Node selection}

Minimum of 3 reference points are required to apply trilatertion technique to find the point of intersection, i.e. the coordinates of OrN. List of Reference Nodes RN consists of all the reference nodes in its communication range and their distance from itself. Unlike in 2D networks [5], selection of reference nodes becomes a difficult task. In 3D networks communication range of each sensor node represents a sphere, Figure.3; hence intersection of 2 spheres gives us a circle in a particular plane, Figure.4. Where as in 2D we are left with only 2 point making the calculations simpler.

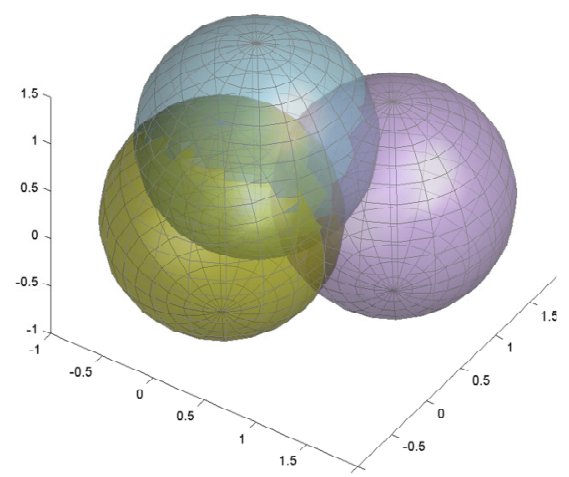

Figure 3. 3D realization of reference nodes

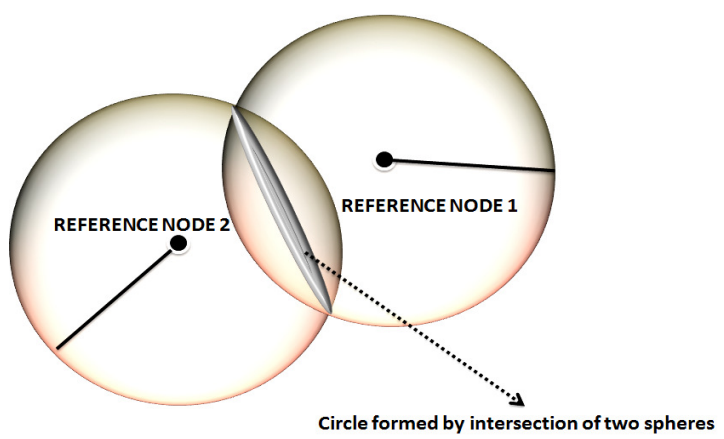

Figure.4. Intersection of two spheres 
The selected RNs should be such that the 3 planes formed by overlapping of the 3 spheres should intersect at a single point.

Consider the equation of the 3 spheres to be:

$$
\begin{aligned}
& \left(\mathrm{x}-\mathrm{x}_{1}\right)^{2}+\left(\mathrm{y}-\mathrm{y}_{1}\right)^{2}+\left(\mathrm{z}-\mathrm{z}_{1}\right)^{2}=\mathrm{R}_{1}{ }^{2} \\
& \left(\mathrm{x}-\mathrm{x}_{2}\right)^{2}+\left(\mathrm{y}-\mathrm{y}_{2}\right)^{2}+\left(\mathrm{z}-\mathrm{z}_{2}\right)^{2}=\mathrm{R}_{2}{ }^{2} \\
& \left(\mathrm{x}-\mathrm{x}_{3}\right)^{2}+\left(\mathrm{y}-\mathrm{y}_{3}\right)^{2}+\left(\mathrm{z}-\mathrm{z}_{3}\right)^{2}=\mathrm{R}_{3}{ }^{2}
\end{aligned}
$$

Where $\left(\mathrm{x}_{1}, \mathrm{y}_{1}, \mathrm{z}_{1}\right),\left(\mathrm{x}_{2}, \mathrm{y}_{2}, \mathrm{z}_{2}\right)$ and $\left(\mathrm{x}_{3}, \mathrm{y}_{3}, \mathrm{z}_{3}\right)$ are coordinates of the $3 \mathrm{RNs}$ and $(\mathrm{x}, \mathrm{y}, \mathrm{z})$ is coordinate of unknown node.

To find the plane in which the intersection of sphere (i.e. a circle) lay, pick one of the equations in (2), (3) or (4) and subtract it from the other two. That will make those other two equations into linear equations in the three variables.

$$
\mathrm{a}_{\mathrm{i}}^{*} \mathrm{x}+\mathrm{b}_{\mathrm{i}}^{*} \mathrm{y}+\mathrm{c}_{\mathrm{i}} * \mathrm{z}=\mathrm{e}_{\mathrm{i}}
$$

These are the equations of the plane in which the intersecting circle lies. Where $\mathrm{i}=1,2,3$.

The intersection of two planes may have many possibilities, depicted in Figure. 5

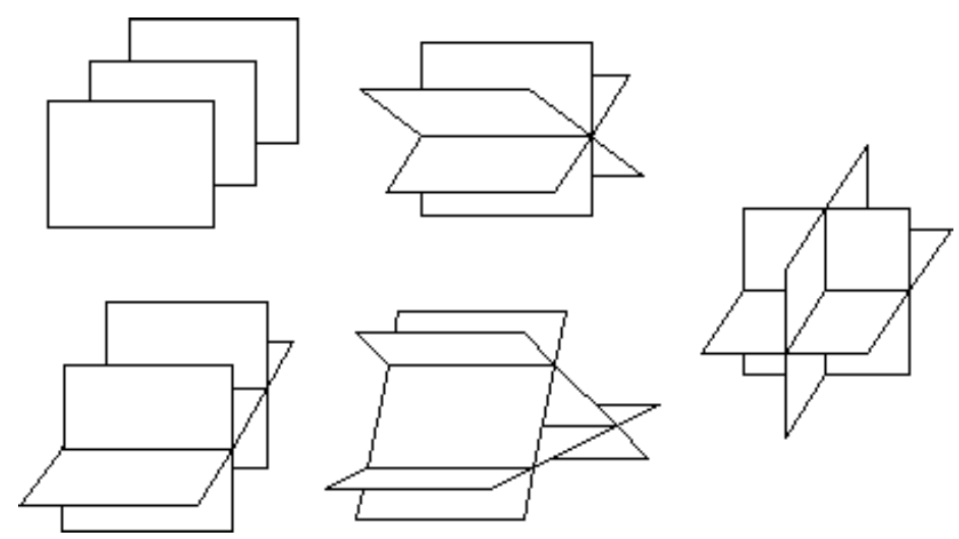

Figure 5. Possibilities of intersection of three planes

To find whether the 3 planes, in (5), meet at a single point it has to satisfy 2 conditions:

(i) The first two planes should not be parallel to each other

(ii) The line formed by the intersection of the first 2 planes should not be parallel to the third

Plane

\subsubsection{Intersection of two planes}

The vectors $\left.\left.<a_{1}, b_{1}, c_{1}\right\rangle=n_{1},<a_{2}, b_{2}, c_{2}\right\rangle=n_{2}$, and $\left\langle a_{3}, b_{3}, c_{3}\right\rangle=n_{3}$ are normal (i.e. perpendicular) to the planes $a_{1} x-b_{1} y+c_{1} z=e_{1}, a_{2} x-b_{2} y+c_{2} z=e_{2}$, and $a_{3} x-b_{3} y+c_{3} z=e_{3}$ respectively. 
In 3D, two planes are either parallel or they intersect in a single straight line $\mathrm{L}$. The planes are parallel whenever their normal vectors $n_{1}$ and $n_{2}$ are parallel, and this is equivalent to the condition that: $\mathrm{n}_{1} \times \mathrm{n}_{2}=0$. When not parallel, $\mathrm{n}_{1} \times \mathrm{n}_{2}>0$ is a direction vector for the intersection line L, Figure 6.

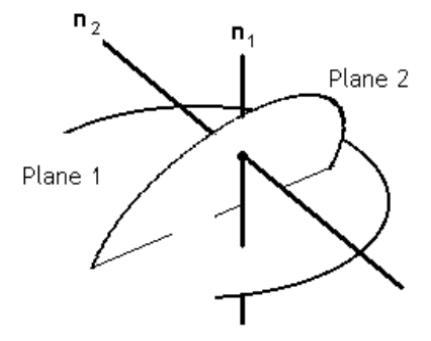

Figure 6. Normal of 2 planes perpendicular to the line of intersection

By simple geometrical reasoning; the line of intersection is perpendicular to both normals. Now, the cross product of these two normal vectors gives a vector which is perpendicular to both of them and which is therefore parallel to the line of intersection of the two planes Equation(6). So this cross product will give a direction vector for the line of intersection and can be used as a reference vector in the direction of the line.

$$
<\mathrm{a}_{1}, \mathrm{~b}_{1}, \mathrm{c}_{1}>\mathbf{x}<\mathrm{a}_{2}, \mathrm{~b}_{2}, \mathrm{c}_{2}>=<\mathrm{a}_{12}, \mathrm{~b}_{12}, \mathrm{c}_{12}>=\mathrm{u}
$$

Where ' $u$ ' is the directional vector of the line formed by intersection of two planes.

\subsubsection{Intersection of a line and a plane}

In $3 \mathrm{D}$, a line $\mathrm{L}$ is either parallel to a plane or intersects at a single point. We first check if $\mathrm{L}$ is parallel to plane by testing if $(\mathrm{n} 3 \bullet \mathrm{u})=0$ which means that the line direction vector $\mathrm{u}$ is perpendicular to the plane normal $\mathrm{n} 3$. If this is true, then the line $\mathrm{L}$ and the third plane are parallel and either never intersects or else L is totally on the plane. If the line and plane are not parallel, then they intersect at a unique point.

\subsubsection{Position estimation}

In UWSN, we assume that the depth of the sensor nodes can be found out by using pressure sensors. How this is done, i.e. the relationship between pressure and depth is explained in [6]. Some percentage of errors involved during calculation of depth with pressure sensors will be highlighted in section 6.2.

Once we are aware about the $3 \mathrm{RN}$ and the depth of the unknown node through pressure sensors, we can find out the position of the unknown node.

Therefore Equation (5) can be written as:

$$
a_{i}^{*} x+b_{i}^{*} y=f_{i}
$$

where $f_{i}=\left(e_{i}+c_{i}^{*} z\right)$ and $i=1,2,3$.

Having with us two variables and three equations we can now solve these equations to get new values of $\mathrm{UN},(\mathrm{x}, \mathrm{y}, \mathrm{z})$. No other data like the distance between the known nodes is required, 
International Journal of Wireless \& Mobile Networks (IJWMN) Vol. 4, No. 2, April 2012

thereby reducing the computational overhead. This is an important inference form our proposed position estimation phase.

\section{ERROR INTRODUCED IN LOCALIZATION ALGORITHM}

\subsection{Effect of ocean parameters on Average Error}

Localization algorithm will work very fine when there are no Distance measurement errors. Distance measurement errors are errors in the distance estimates between the non localized node and references. Distance measurement is highly influenced by Sound speed, which in turn depends on temperature, salinity and depth. Hence distance calculated is directly influenced by speed of sound, as in equation (1) [8] [9].

We will be using a new equation proposed by Leroy et al [10] for the calculation of sound speed in seawater which is a function of temperature, salinity, depth, and latitude in all oceans and open seas. Equation (8) shows the relation between underwater parameters and the sound speed. Where T: Temperature, S: Salinity, Z: Depth and $\phi$ : Latitude.

$$
\begin{aligned}
& \text { Speed of sound ' } \mathrm{C} \text { ' }=1402.5+5 \mathrm{~T}-5.44 \times 10^{-2} \mathrm{~T}^{2}+2.1 \times 10^{-4} \mathrm{~T}^{3}+1.33 \mathrm{~S}-1.23 \\
& \mathrm{X} 10^{-2} \mathrm{ST}+8.7 \times 10^{-5} \mathrm{ST}^{2}+1.56 \times 10^{-2} \mathrm{Z}+2.55 \times 10^{-7} \mathrm{Z}^{2}-7.3 \times 10^{-12} \mathrm{Z}^{3} \\
& +1.2 \times 10^{-6} \mathrm{Z}(\Phi-45)-9.5 \times 10^{-13} \mathrm{TZ}^{3}+3 \times 10^{-7} \mathrm{~T}^{2} \mathrm{Z}+1.43 \times 10^{-5} \mathrm{SZ}
\end{aligned}
$$

When we calculate the distance between the RN and the Ordinary Node ON, owing to special mechanical properties of sea water, the sound moves at a mean speed around $1500 \mathrm{~m} / \mathrm{s}$. Sound travels faster with increase in T,S,P. Temperature strongly effects the speed of sound (i.e. soundtravels faster in warm water than in cold water). Hence we assume that the speed of acoustic signal will be $1500 \mathrm{~m} / \mathrm{s}$. as in equation (9).

$$
\mathrm{d}_{1}=\mathrm{S}_{1} * \mathrm{t}
$$

But as we have seen earlier that the speed always varies with the change in oceanographic parameters like temperature, Salinity and depth at which the node is placed. The new distance with errors can now be calculated as in equation (10).

$$
\mathrm{d}_{2}=\mathrm{S}_{2} * \mathrm{t}
$$

Where, $S_{1}$ is considered to be $1500 \mathrm{~m} / \mathrm{s}$ and $S_{2}$ is the speed of sound calculated for varying temperature, salinity and Depth values. Here $t$ will remain same as the time taken will be calculated using TOA method. When network consist of $\mathrm{N}$ sensors then any nodes constellation can be fully described as $\mathrm{N}$ by $\mathrm{N}$ matrix. Elements of this matrix dij equal to distance between neighbor nodes $i$ and $j(i, j=1 \ldots N),-1$ if nodes $i$ and $j$ are too far to communicate and 0 if $j=i$. To find node $i$ position it is necessary to know at least three dij $>0$ elements where $j=1$...N with $j \neq i$. Communication range $R$ has to be greater than the distance dij. For simulation purpose we propose an equation (11) to calculate the erroneous distance.

$$
\operatorname{dij}^{*}=\left\{\begin{array}{cc}
\operatorname{dij}((\mathrm{d} 2 / \mathrm{d} 1)-1) & \text { when } \operatorname{dij}<=\mathrm{R} \\
-1 & \text { when } \operatorname{dij}>\mathrm{R}
\end{array}\right.
$$


The above mentioned technique was simulated for 2D UWSN [13] and is also suitable for 3D scenario. This technique was analyzed for varying sound speed caused due to ocean parameters. The analysis concludes that the sound speed was predominantly influenced by temperature and very less by salinity and pressure [13]. As in Figure.7:

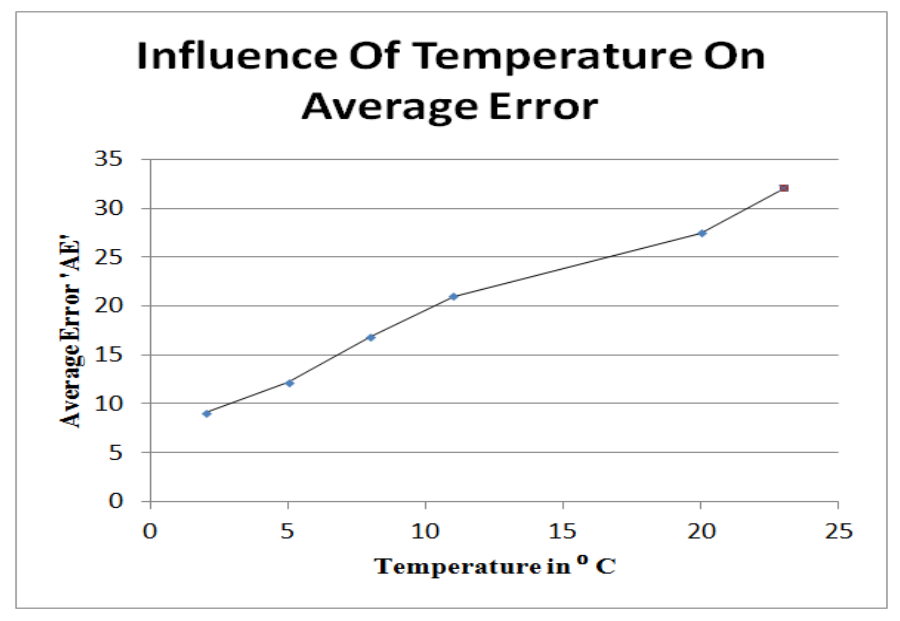

Figure 7. Influence of Temperature on Average Error in 2 D Scenario

Sound speed was predominantly influence by temperature rather than salinity and pressure [11]. Hence for analysis of effect of sound speed on Localization algorithm in 3D scenario, we will keep salinity and pressure as a constant factor and vary temperature to calculate its impact on calculated node location.

\subsection{Effect of Depth measurement on Average Error}

Apart from Temperature, Salinity and Pressure being the major factors influencing the errors in distance estimation, Depth (calculated using pressure sensors) also influences the average error in node localization.

Useful guidance and suitable equations for converting pressure into depth and depth into pressure can be found in [6]. The key equation for Conversion of pressure into depth is:

$$
Z_{s}=\frac{9.72659 \times 10^{2} P-2.512 \times 10^{-1} P^{2}+2.279 \times 10^{-4} P^{3}-1.82 \times 10^{-7} P^{4}}{g(\phi)+1.092 \times 10^{-4} P}
$$

where $\mathrm{g}(\varnothing)$ is given as the international formula for gravity.

These equations are based on the algorithm of UNESCO 1983 [12].

\section{EXPERIMENTAL RESULTS}

We propose a localization algorithm which can efficiently work for 3D UWSNs. In our simulation 500 nodes were deployed over an area of $1000 \times 1000 \times 1000$ meters. The communication range of every sensor node ' $R$ ' was taken between 250 to be meters. The initial RN were place at $(1,100,50),(100,100,1)$ and $(100,1,100)$ respectively. Certain assumptions 
International Journal of Wireless \& Mobile Networks (IJWMN) Vol. 4, No. 2, April 2012

made were, all transmitters and receivers in the system were precisely synchronized and only nodes with beacon flag as ' 1 ' can act as RN's. Nodes are deployed randomly to form a 3 Dimensional architecture. The sensor nodes were placed at varying depth. Inorder to compute accurate results simulations were run for 50 simulations to take average results for different underwater parameters.

The UW parameters discussed in section 6.1 and 6.2 were incorporated in the simulation to find the deviation from the actual location of a sensor node.

The proposed localization algorithm was simulated to analyze the effect of temperature on sound speed and indeed on Average error. The influence of depth measured using pressure sensors was also incorporated to find the possibilities of errors induced.

For Temperature being the major factor influencing the estimated distance, in our simulation we take temperature as a variable and keep salinity and depth as constant values.

In Figure.8. Salinity $=35 \%$, Latitude $=60^{\circ}$ and Temperature varies from $2-23{ }^{\circ} \mathrm{C}$

\section{Influence of Sound Speed on Proposed}

3D Localization Algorithm

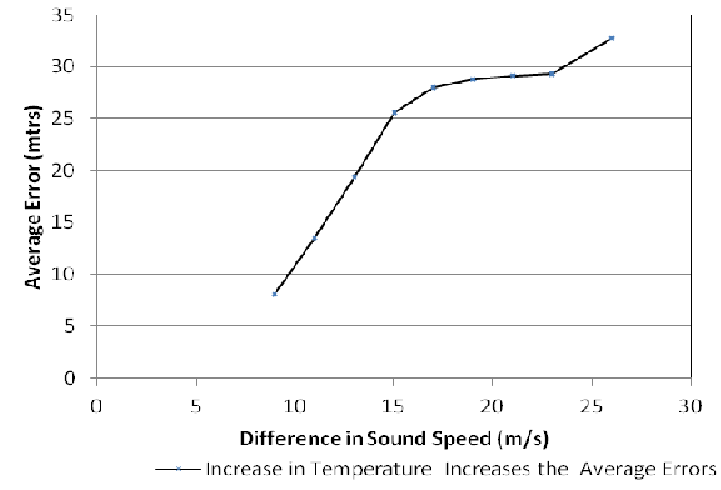

Figure 8. Average error induced by temperature varing from $\left(2-23{ }^{\circ} \mathrm{C}\right)$

Figure.8. shows that the temperature is the dominating factor which influences the localization results. In our simulation the maximum difference between the actual and calculated distance was 40 metres, when temperature varied from $2-23{ }^{\circ} \mathrm{C}$.

The erroneous distance calculation for depth as a function of pressure is as shown in Equation (12). 


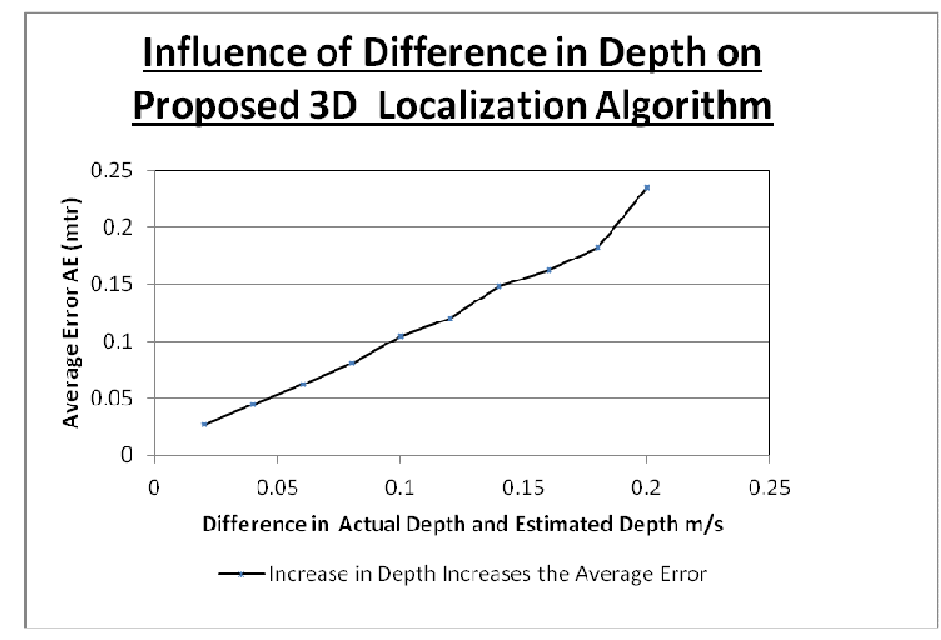

Figure 9. Average error induced by change in calculated depth. (0.02 to $0.2 \mathrm{~m} / \mathrm{s})$

Depth as compared to temperature is not a dominant parameter in location calculation, but must be considered as a active participant in localizing a sensor node. Simulation was carried out for difference in depth varing form 0.02 to $0.2 \mathrm{~m} / \mathrm{s}$. Figure.9. shows the influence of difference in depth on proposed 3D Localization algorithm.

\section{Conclusion}

We propose a distributed self organizing algorithm for UWSN. UW networks experience various impediments. Factors like temperature, salinity and pressure in UW which influence the UWSN localization were analysed. We studied the effect of errors caused due to variation in sound speed and depth calculated using pressure sensors. Our proposed algorithm caters for and unobtrusive service for UW. Our proposed localization technique incorporates likely errors anticipated while operating underwater for Large scale UWSN. The proposed algorithm works efficiently, with an error not more than 33 meters for temperature ranging between 23 to 26 degrees centigrade and error of approximately 0.23 meters using difference in estimated depths. The estimated location of the unknown node can deviate not more 40 meters when exposed to varying temperature and pressure parameters.

\section{REFERENCES}

[1] Ian.F.Akyildiz, Dario Pomplili and Tommaso Melodia, -Underwater acoustic sensor net-works research challengesll,sciencedirect, 2005.

[2] Melike Erol, Hussein T and Sema Oktug, A Survey of Architectures and Localization Techniques for Underwater Acoustic Sensor Networks, IEEE communications and survey \& tutorials, 2011.

[3] Hui Liu , Survey of Wireless Indoor Positioning techniques and systems, IEEE transactions on systems, man, and cybernetics-part c: applications and reviews, vol. 37, no. 6,2007

[4] K. H. Lee, C. H. Yu, J. W. Choi and Y. B. Seo, ToA based Sensor Localization in Underwater Wireless Sensor Networks, SICE Annual Conference 2008.

[5] Samedha Naik and Manisha J Nene, Self Organizing Localization Algorithm for Large Scale Underwater Sensor Networksll, IEEE International Conference on Recent Advances in Computing and software systems, 2012, In Press.

[6] Claude C. Leroy, Depth-pressure relationships in the oceans and seas, Journal of the acoustical Society of America,103 (3), 1998 
International Journal of Wireless \& Mobile Networks (IJWMN) Vol. 4, No. 2, April 2012

[7] Kang Hoon Lee, Chang Ho Yu, Jae Weon Choi and Young Bong Seo, - ToA based Sensor Localization in Underwater Wireless Sensor Networksl, SICE Annual Conference 2008.

[8] Xavier Lurton, -An Introduction to Underwater Acoustics, Principles and Applications\|, Book, Springer Publication,2010.

[9] M. M. Ali, Sarika Jain and Radhika R, Effect of Temperature and Salinity on Sound Speed in the central Arabian Sea, The Open Ocean Engineering Journal, 4, 71-76, 2011.

[10] C.C Leroy, Stephen P, -A new equation for the accurate calculation of sound speed in all Oceans", Journal of the acoustical Society of America,2774-2782.,2008

[11]S Jamshidi and Md. N. B. Abu, An Analysis on Sound Speed in Seawater using CTD Data, Journal of applied sciences, 10(2): 132-138,2010.

[12]N. P. Fofonoff and R.C. Millard, Algorithms for computation of fundamental properties of sea water, Unesco technical papers in marine science, 1983.

[13] Samedha Naik and Manisha J Nene, Effect of sound speed on localization algorithm for underwater sensor networks, International Conference on Wireless, Mobile networks and Applications (WiMoA),2012. In Press.

[14] Jim Partan, Jim kurose and Brain Neil, A Survey of Practical Issues in Underwater Networks WUWNet' 06, 2006.

[15] Low Tang Jung and A.B.Abdullah, Underwater Wireless Network, International Conference on Electrical, Control and Computer Engineering, IEEE 2011

[16]Vijay Chandrasekhar, Winston KG, Yoo SC and How VE, Localization in Underwater Sensor Networks- Survey and Challenges, 2006, WUWNet'06.

[17]Nor-Syahidatul N.Ismail, Liban Abdullahi Hussein, Sharifah H.S.Ariffin, “Analyzing the Performance of Acoustic Channel in Under Water Wireless Sensor Network (UWSN), Fourth Asia International Conference on Mathematical/Analytical Modelling and Computer Simulation,2010.

[18]D.Mirza and C. Schurgers, Motion-aware self-localization for underwater networks, San Francisco, California, USA,2008, pp. 51-58.

[19]Y. Zhou, B. Gu, J.C.K Chen and H. Guan, An range-free localization scheme for large scale underwater wireless sensor networks, J. Shangai Jiaotong University, vol. 14 no.5 ,pp 562-568,2009.

[20]D Mirza and C Schurgers, Collaborative localization for fleets of underwater drifters, IEEE Oceans, Vancouver, BC,2007

[21] M. Erol, Luiz F. M and M. Geria, "Localization with Dive'N'Rise (DNR) Beacons for Underwater Acoustic Sensor Networks", 2007, WUWNet'07

[22]K. Chen, Y Zhou and J He, A localization scheme for underwater wireless sensor networks, International Journal of Advanced Science and Technology, vo. 4, 2009.

[23] Melike Erol and Luiz Filipe, -AUV-Aided Localization for Underwater Sensor Networks\| 2007, WASA

[24]H.Luo, Z Guo, W Dong and Y Z Hong, Ldb: Localization with directional beacons for sparse 3d underwater sensor networks, Journal of Networks, vol. 5 no.1, 2010

[25]M. Erol, Luiz F.M, Antonio C, Multi Stage Underwater Sensor Localization Using Mobile Beacons, The Second International Conference on Sensor Technologies and Applications,IEEE,2008.

[26]Z Zhou, J Cui and S Zhou, Localization for large-scale underwater sensor networks, Poc. IFIP Networking, Atlanta, Georgia,USA, May 2007,pp 108-119

[27]M T Isik, O.B Akan, A three dimensional localization algorithm for underwater acoustic sensor networks, IEEE Trans. Wireless Communication, vol. 8, no.9.pp4457-4463.

[28]J Cui, Z Zhou and A Bagtzoglou, Scalable localization with mobility prediction for underwater sensor networks, in Proc. Second workshop on Underwater networks (WuWNet), Montreal, Quebec, ananda,2007.

[29] Yi Zhou, K Chen, J He ,J Chen and Alei L, “A Hierarchical Localization scheme for Large Scale Underwater wireless sensor networks ",2009, 11th IEEE International Conference on High Performance Computing and Communications. 
International Journal of Wireless \& Mobile Networks (IJWMN) Vol. 4, No. 2, April 2012

\section{Authors}

Samedha S Naik received the bachelor of engineering degree in Computer Engineering from Goa University, India. Currently she is perusing her M.Tech degree in Computer Engineering from Defence Institute of Advanced Technology. DIAT, Pune, India. Her research interests are in communication protocols, programming languages and underwater sensor network

Manisha $\mathbf{J}$ Nene is currently associated with the Department of Applied Mathematics and Computer Engineering in Defence Institute of Advanced Technology, India. Her areas of interest and research are Modelling and Simulation, Wireless Sensor Networks and High Performance Computing. 